\title{
A Fixed-Based Flight Simulator Study: The Interdependence of Flight Control Performance and Gaze Efficiency
}

\author{
Lewis L. Chuang ${ }^{1}$, Frank M. Nieuwenhuizen ${ }^{1}$, and Heinrich H. Bülthoff ${ }^{1,2, \star}$ \\ 1 Department of Perception, Cognition and Action, \\ Max Planck Institute for Biological Cybernetics, Tübingen \\ 2 Department of Cognitive and Brain Engineering, Korea University \\ \{lewis.chuang, frank.nieuwenhuizen, \\ heinrich.buelthoff\}@tuebingen.mpg.de
}

\begin{abstract}
Here, a descriptive study is reported that addresses the relationship between flight control performance and instrument scanning behavior. This work was performed in a fixed-based flight simulator. It targets the ability of untrained novices to pilot a lightweight rotorcraft in a flight scenario that consisted of fundamental mission task elements such as speed and altitude changes. The results indicate that better control performance occurs when gaze is more selective for and focused on key instruments. Ideal instrument scanning behavior is proposed and its relevance for training instructions and visual instrument design is discussed.
\end{abstract}

\section{Introduction}

Like driving a car, piloting a rotorcraft is a closed-loop motor control task that relies heavily on visual feedback. Visual feedback informs us on the outcome of our control inputs and whether it is necessary to change these inputs in order to arrive at our desired performance levels. Information such as velocity is available from the outside world, it can also be provided more precisely via instruments (eg., airspeed indicator).

Piloting a rotorcraft is, arguably, more difficult than driving a car for several reasons. First, it offers movement in more degrees of freedom. A rotorcraft pilot is required to control not only the vehicle's airspeed and heading, but also its altitude. Second, the control devices of a rotorcraft are not directly mapped to the direction of the vehicle's motion. In a car, accelerations and decelerations can be achieved by exerting pressure on the accelerator and braking pedal, respectively. Maintaining a constant velocity is achieved by determining the appropriate pressure to consistently apply to the accelerator. This connection between control

\footnotetext{
* The work in this paper was supported by the myCopter project, funded by the European Commission under the 7th Framework Program.
} 
device and vehicle control is direct and intuitive. In contrast, rotorcraft acceleration is achieved by tilting the rotor disc (and thus, the helicopter) forward. Lateral decelerations are effected by tilting the rotor disc in the opposite direction. Thus, flying at a constant airspeed requires continuous adjustments of the rotor disc's tilt. Last but not least, rotorcraft controls are coupled. Tilting the rotor disc forward will not only accelerate the rotorcraft in the same direction but will also reduce its upward thrust, hence causing a loss in altitude. Conversely, decelerating the rotorcraft by reducing the forward tilt of the rotor disc will increase the upward thrust, causing an increase in altitude.

Efficient monitoring of available visual feedback information can be expected to be relevant to effective rotorcraft control. By moving his eyes, the pilot is able to switch between monitoring several visual feedback cues (eg., airspeed, altitude, heading, etc.) and checking for any discrepancies between desired and actual values. This underlies his ability to manipulate the rotorcraft's controls appropriately, such as to reduce detected discrepancies. Scanning strategies have also been shown to differ across levels of control expertise. Recently, navigation accuracy was found to negatively correlate with the amount of time that pilots looked at the "outside" world [1. Thus, expertise might be reflected in a decreasing reliance on outside world cues.

Gazetracking methods allow researchers to determine how visual feedback information is sought after during flight control. In the context of driving and aviation, gazetracking data can identify which visual cues and instruments are relied on the most. For example, the primary flight display was the cockpit instrument that pilots looked at the most during the approach and landing of a A330 fixed wing aircraft - namely, $40 \%$ of looking time 2. Within this, attention was particularly dedicated to information relating to the attitude of the plane, its airspeed and altitude. Findings such as these can improve cockpit designs, by tailoring the visualization of information to fit the pilot's mission objectives.

To date, relevant eye-tracking research has predominantly addressed expert flight performance. Relatively little is known about the relationship between flight control performance and instrument gaze behavior in novices. Our research seeks to identify methods and technologies that will enable personal aviation transport, by allowing flying a personal aerial vehicle to be as easy as driving a car [3]. To do so, it is necessary to measure and model the behavior of untrained individuals that underlie their ability to control an aircraft.

In this study, we designed a flight scenario that reflected a daily commute from a suburban area to a city region. Novices were required to pilot a lightweight rotorcraft model in a helicopter flight simulator that was equipped with a remote eye-tracking system. The motivation of this work was to explore the relationship between flight control performance and scanning strategies of novices. Specifically, we investigated how novices with varying levels of control performance might differ in terms of how they looked at their flight instruments for control information. It should be mentioned that flight trainees are explicitly taught strategies on how to scan their flight instruments for flight control. 
In this paper, we investigate how novices are likely to monitor flight instruments without having received such instructions.

The remainder of this paper is organized as follows. Section 2 provides a detailed description of the flight mission and the fixed-base simulator environment. It also describes how data was collected for subsequent analyses. Section 3 presents the descriptive statistics of our current work and our interpretations. Section 4 discusses the implications of the current results and how that could influence training instructions and the interface design of visual instrumentation.

\section{Methods}

\subsection{Participants}

Four male participants were recruited for this study (age range: 29-34 years). They possessed normal or corrected-to-normal vision. None of the participants had formal flight training for rotorcraft. More importantly, they have never received any formal instructions for instrument scanning strategies.

\subsection{Apparatus and Flight Model}

The fixed-based flight simulator was based on a simulated model of a light-weight helicopter with a large bandwidth for control inputs [5] (Figure 11). Visualizations of the outside world were based on the topography of San Francisco, USA. These were presented via a large multi-panelled display that rendered a world environment (field-of-view: $105^{\circ}$ x $100^{\circ}$ ).

A heads-down display consisted of eight standard flight instruments that indicated the rotor speed (RS), airspeed (ASI), attitude (AI; also referred to as the artificial horizon), altitude (AT), torque (TQ), compass (CO), heading (HI), vertical speed (VSI) — see Figure 1 (inset). These are arranged in the standard T-arrangement of ASI, AI, AT on the second to four position in the top row, and $\mathrm{HI}$ in the third position of the bottom row. Eye-movements on the instrument panel were recorded using a $60 \mathrm{~Hz}$ remote eyetracker (faceLAB; SeeingMachines). The users gaze vector was estimated up to an accuracy of $1.5^{\circ}$, which was sufficient for determining the specific flight instrument that was fixated by the user.

A cyclic stick, collective lever and foot-pedals comprised the control devices. The cyclic stick controls the tilt of the rotorcraft. Namely, the rotorcraft will tilt in the same direction as the cyclic stick. If the cyclic is moved forward, the rotorcraft tilts forward; if the cyclic is moved aft, the rotorcraft tilts aft, and so on. These changes are reflected in the attitude indicator (AI), which indicates the rotorcraft's relation to the horizon - that is, whether it is pointing below or above and whether it is level with the horizon. Tilting the rotorcraft such that it points below the horizon will increase airspeed (ASI). This forward tilt will induce a loss in altitude (AT) unless accompanied by an increase in thrust power (RS). Conversely, reducing airspeed (ASI) by decreasing the rotorcraft's forward 


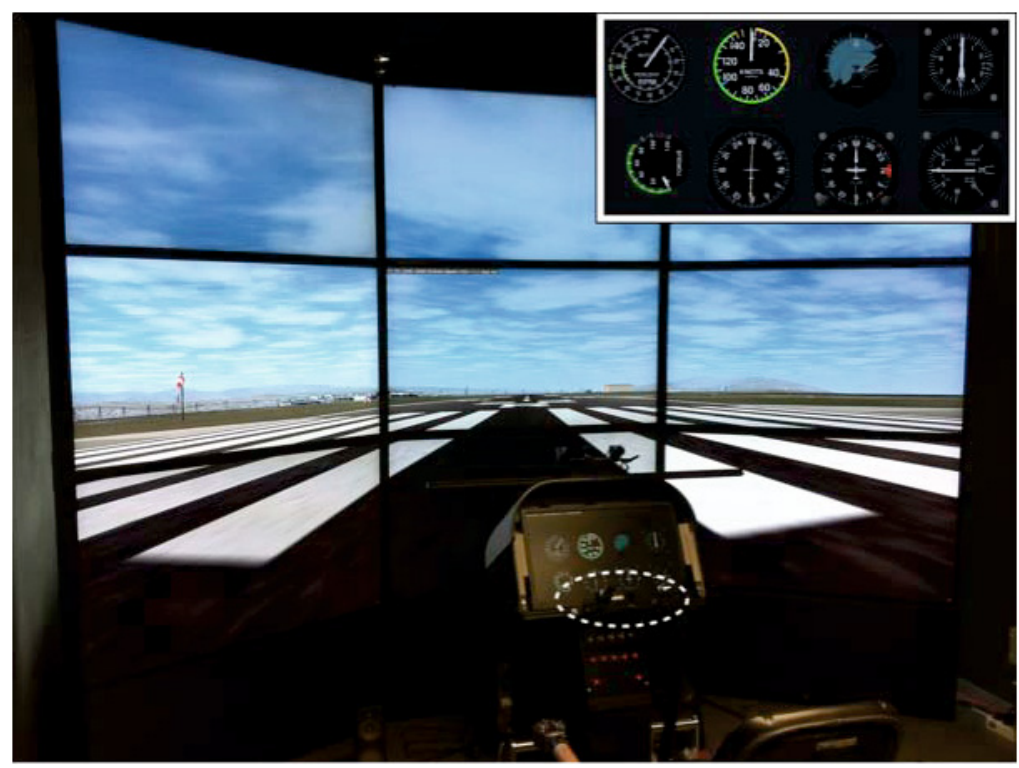

Fig. 1. Flight simulator with remote eye-tracking cameras (indicated in the white ellipse). Instrument panel is featured inset (From left to right. Top panel: RS, ASI, AI, AT. Bottom panel: TQ, CO, HI, VSI.)

tilt will induce an elevation in altitude if the thrust (RS) is not simultaneously reduced. Control of thrust is determined by the collective lever. The yaw of the rotorcraft can be controlled by the foot-pedals. This compensates for any undesired torque forces (TQ) on the fuselage that result from changes in the rotor speed (RS). The global bearing of the rotorcraft is indicated by the compass (CO) and its current heading relative to its destination by a heading indicator (HI).

\subsection{Flight Scenario}

Prior to experimentation, participants had at least 10 hours of experience in the flight simulator, which familiarized them with the dynamics of the simulated vehicle and the instrument layout. The participants were aware of how the controls were coupled and how to use them in combination to achieve simple maneuvers — such as to accelerate, decelerate, ascend and descend — without creating large instabilities.

Four experimental sessions were conducted per participant with a common flight mission. Our participants were to fly from a suburban airfield to the city area of San Francisco, USA. This reflects a daily personal aerial vehicle commuting scenario. Three classes of mission task elements (MTEs) comprised the entire flight scenario: straight and level flight, altitude change, and airspeed change. 
This flight scenario was designed, with the assistance of an experienced helicopter pilot (approx. 650 flight hours), to be within the control capabilities of a beginning trainee.

\subsection{Data Collection}

The airspeed and altitude of the rotorcraft were recorded during each flight session, in tandem to our participants' gaze. This allowed us to characterize flight control performance (see Subsection 3.1)

Nine regions of interest (ROI) were designated. Namely, the eight instruments and the outside world. Dwells were defined as periods when the participants' gaze intersected with one of these ROIs. Gaze data across the flight mission were first classified into those that belonged to the heads-down instrument panel or the world environment. Dwells belonging to the instrument panel were further classified for the specific instruments using a k-nearest-neighbor filter. Thus, it was possible to derive the number of dwells per ROI as well as the likelihood that a ROI dwell follows from another ROI dwell (see Subsection 3.2).

\section{Results and Discussion}

\subsection{Flight Control}

The current flight scenario required our participants to demonstrate control over their airspeed and altitude. Figure 2 (upper half) illustrates the desired trajectory for these two attributes and how our participants deviated from them across their final flight mission. Performance in keeping altitude and airspeed was determined by calculating the root-mean-squared error (RMSE) between desired and actual value. For MTEs in which a change in altitude or airspeed was performed by the participants, the RMSE for altitude error or velocity error, respectively, was not determined.

All participants showed increased instabilities in keeping altitude with respect to straight-and-level flight after they performed changes in airspeed (acceleration and deceleration). Conversely, changes in altitude did not result in more airspeed instability with respect to straight-and-level flight.

Across the whole flight mission, our participants differed primarily on their ability to control airspeed, rather than altitude (see Fig. 2, lower half). Across all participants, a prominent increase in altitude control difficulty is observed in the later stages of the flight mission, especially when decelerating and descending.

We used the RMSE metric to rank-order the performance of our participants, such that we could evaluate their gaze behaviour for corresponding trends. Participant S4 performed worst in keeping airspeed and altitude, whereas participants S1, S2 and S3 had similar performance in keeping altitude, but showed decreasing performance in keeping airspeed, respectively. Henceforth, they will be referred to as S1, S2, S3 and S4 in increasing order of RMSE, and color-coded in the figures as dark-green, green, red and dark-red respectively. 


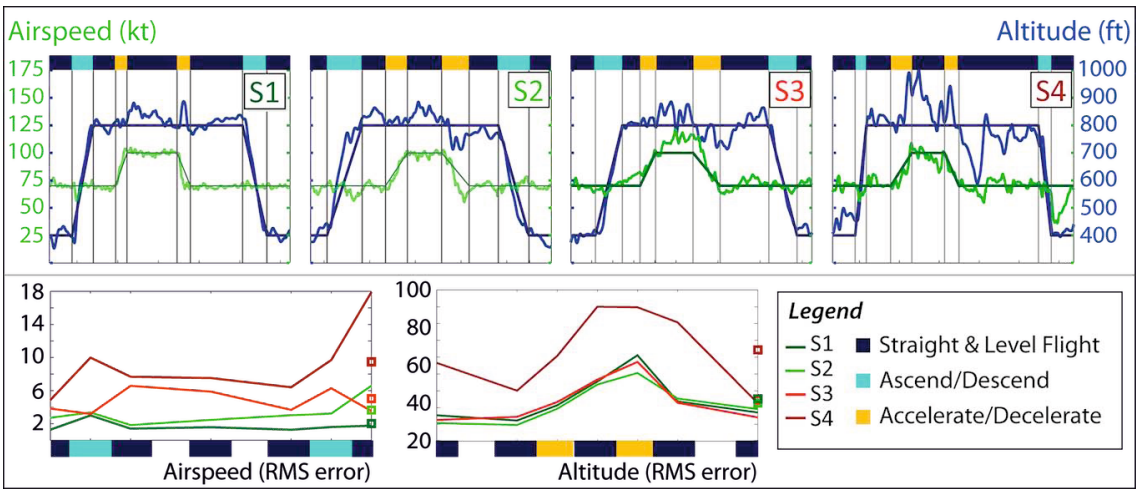

Fig. 2. Upper half: Control performance during a standard flight scenario. Participants are ranked according to decreasing control performance, from left to right. Nine mission task elements comprise the flight scenario: straight and level flight (dark blue), altitude change (light blue), airspeed change (yellow). Desired values for airspeed (green) and altitude (blue) across the flight scenario are indicated by the underlying straight lines and actual performance is overlaid. Lower half: Mean RMSEs of flight control performance of each participant. The mean RMSEs across flight mission are indicated on the right vertical axis.

\subsection{Gaze Behavior}

Gaze Selectivity across Instruments. Figure 3 illustrates the mean number of dwells on each instrument and the outside world (rows) for each MTE (columns) across the flight mission. Each grayscale histogram reflects the number of times each instrument was looked at; white denotes the maximum number of dwells within each individual histogram and black indicates that the instrument was never looked at.

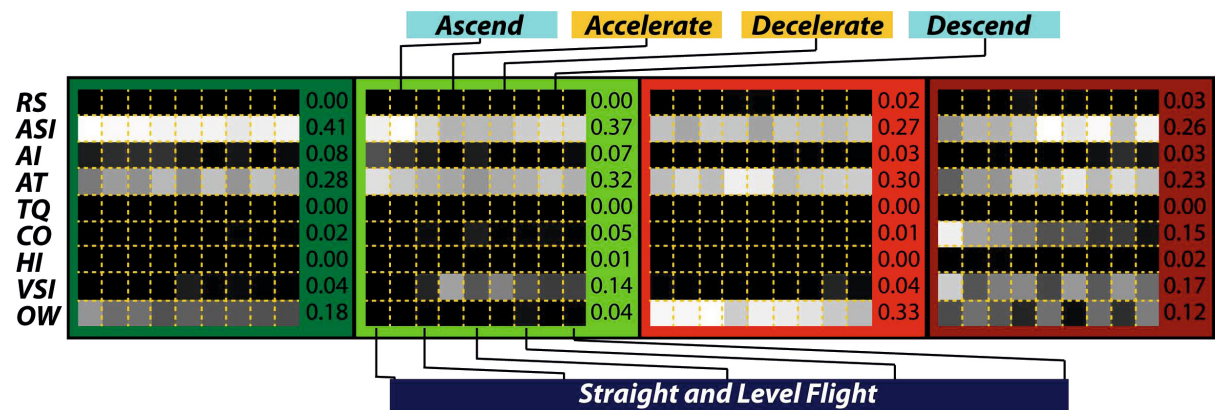

Fig. 3. Grayscale histogram for the mean number of dwells on each instrument and outside world (rows), for each maneuver (columns). The mean proportions of instrument dwells are reported on each histogram's right. 
Every participant consistently demonstrated a gaze preference for the airspeed indicator (ASI) and altimeter (AT). This corresponds with the main determinants for flight control performance in our flight scenario. This instrument preference did not vary according to the changing mission task elements.

To reiterate, our participants primarily differed in terms of their ability to control airspeed, rather than altitude. A corresponding trend is noted in the gaze allocation to instruments. Mean proportion of dwells on ASI decreased from S1 to S4. Mean proportion of dwells on AT did not vary much between S1, $\mathrm{S} 2$ and S3 but was noticeably less for S4.

More than $85 \%$ of S1's dwells can be attributed to ASI, AT and the outside world $(\mathrm{OW})$, in decreasing order of preference. This can be contrasted with S4's gaze, whereby the same three instruments only accounted for $61 \%$ of dwells. In comparison, S4 was less selective than S1 and looked at the compass (CO) and vertical speed indicator (VSI) more often than S1, at the expense of his monitoring levels of ASI and AT.

An interesting contrast can be observed between S2 and S3. S2 does not look at the OW as often as S3. In fact, S3 looks at the OW most frequently, relative to the other participants. Instead, S2 demonstrates a gaze preference for the VSI, especially during the middle of the flight mission from acceleration to deceleration, when control performance is most vulnerable across all participants (see Fig. 2).

Looking at Instruments versus Outside World. Control performance in novice pilots is unlikely to be characterized by the relative distribution between looking at the instruments and the OW. About $90 \%$ of the dwells on instruments prior to looking at the OW could be accounted for by 8 or fewer dwells in S1's flight missions. And in S2, S3 and S4s' flight missions by 22, 3 and 15 dwells or fewer.

Instrument Scanning. Given that a particular instrument is being looked at, what are the other instruments that will be looked at next? Figure 4 illustrates these gaze relationships between instruments. Each column indexes an instrument and reflects the number of subsequent dwells on other instruments (rows).

The obvious relationship is that between ASI and AT. For S1, he is equally likely to look at AT and OW after looking at ASI when ascending and accelerating. When his control performance decreases during decelerating and descending, he looks at the OW less frequently and switches gaze primarily between AT and OW. Notably, S1 treats ASI as the primary reference point in instrument scanning. That is, S1's gaze consistently returns to this instrument after referencing AT and OW. Dwells in AT and OW tend to occur after ASI dwells (see column 2 for S1) and, reciprocally, dwells in AT and OW tend to be followed predominantly by ASI dwells (see columns 4 and 9 for S1).

A similar pattern is observed for S2. Unlike S1, however, S2 either switches gaze between ASI and AT almost exclusively (ascend, descend) or between ASI, AT and VSI (accelerate, decelerate). He does not exhibit a primary instrument that he returns to preferentially after referencing other instruments, like ASI in S1's case. 


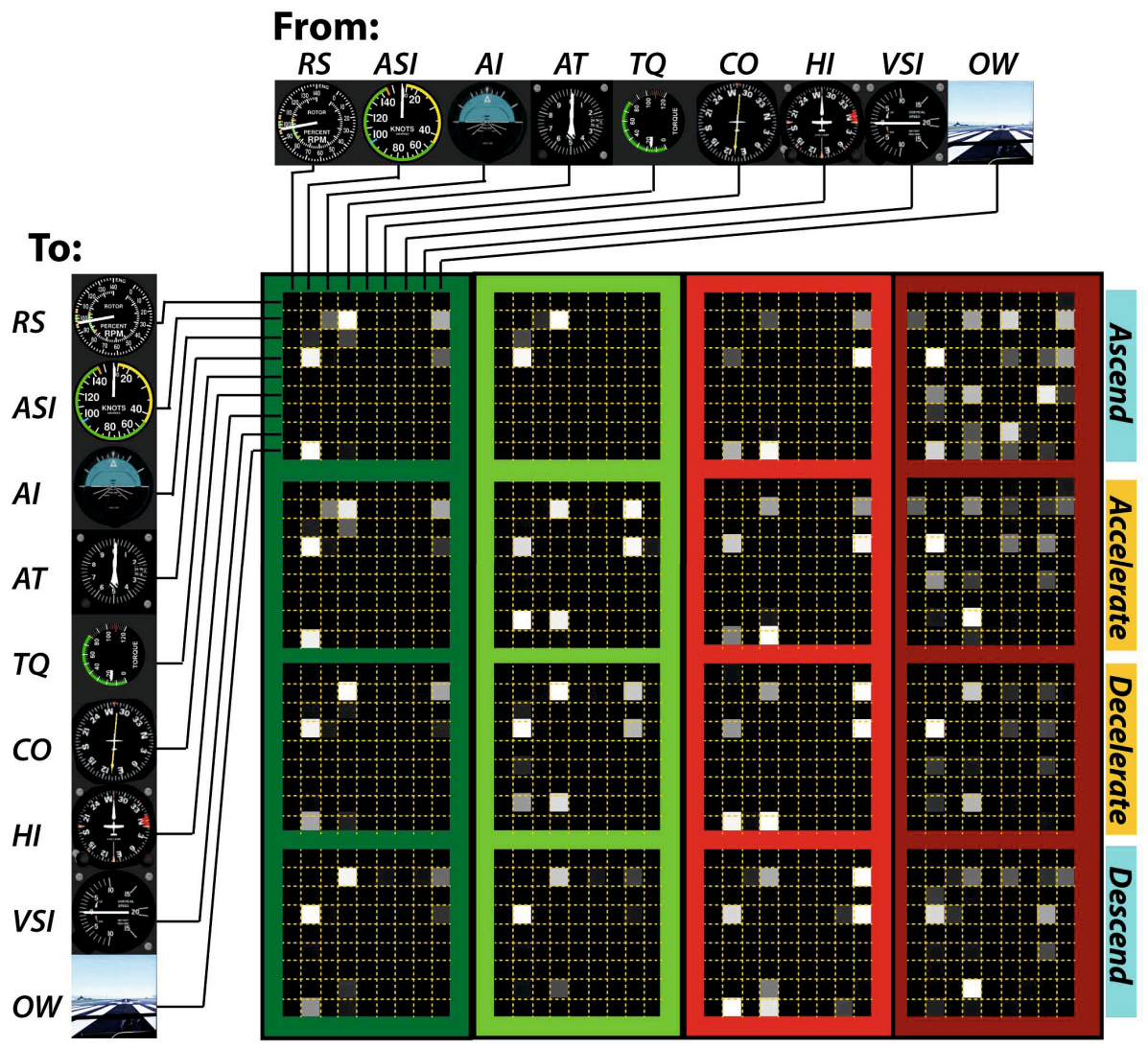

Fig. 4. Transition matrix of instrument dwells in grayscale, for the key maneuvers. Each column is indexed by an instrument. White denotes the maximum number of subsequent dwells within each matrix.

S3's gaze alternates between ASI, AT and OW. Unlike S1, S3 relies on the OW instead of ASI as the primary reference point. In contrast to both S1 and S2, S3 demonstrates less turn-taking between ASI and AT. It is also interesting to note that this reliance on $\mathrm{OW}$ as a primary reference point increases when control performance deteriorates (ie., decelerate, descend).

There is no clear transitional pattern of gaze turn-taking across the instruments for S4. The lack of a consistent gaze strategy could underlie S4's poor control performance.

\section{Conclusions}

The current paper presents a descriptive study that was motivated to demonstrate and understand the relationship between flight control performance and 
gaze behavior. Our findings show that gaze behavior varies with control performance. In particular, participants with better control performance exhibited gaze behavior that was more selective for key instruments. Our best performer demonstrated a selective and ranked preference for different visual information, whilst our second and third performer distributed gaze equally across three different sources of visual information. The worst performer distributed his gaze across five sources of visual information.

The current findings do not allow us to conclude that selective gaze patterns generate good flight control performance (or vice versa). Nonetheless, we can recommend training instructions for novice pilots with regards to instrument scanning behavior. This can be expected to yield improvements given that gaze strategies are easier to be voluntarily controlled by novice pilots than flight control expertise. Fundamentally, instrument scanning should always be centered on the instrument that is key to control performance. This means that eye-movements should ideally return to one key instrument after referencing secondary sources of information. Distributing gaze evenly and across many instruments without such an underlying strategy is ill-advised. Indeed, such instructions are provided in formal flight training; the T-arrangement is intended to encourage such instrument scanning behavior.

The current work was intended to understand how novices without formal flight instructions would scan instruments. It is interesting to note that they generally prioritized the airspeed indicator, followed by the altitude indicator. This suggests that novice pilots intuitively react to changes in their airspeed and altitude and compensate accordingly. In formal training, pilots are instructed to treat the attitude indicator as the key instrument, given that it is the rotorcraft's attitude that dictates current airspeed and the need to adjust for changes in the altitude. In this regard, formal training serves the function of training novices to behave in a more anticipatory and less reactive fashion. This could account for better control performance and reduced workload.

A principled approach for diagnosing gaze selectivity in trainees could be useful in determining the skill levels of novices (cf. 1] ). In fact, flight instructors sometimes rely on playbacks of eye-movements to identify inappropriate scanning strategy in trainee pilots [6]. The current analytical approach is one possible approach for quantifying gaze selectivity.

Finally, the spatial layout of visual instruments could be designed to encourage optimal scanning strategies. First, selective attention to relevant information could also be encouraged by removing the clutter of non-relevant instruments. Second, the spatial layout of instruments could emphasize the importance of a primary instrument by placing it in a central position. This could encourage users to return to it after referencing other instruments (cf., S1's scan pattern), rather than to treat every instrument equally.

Future work should be directed towards identifying effective scanning strategies for different maneuvers and key objectives. In the current work, constant airspeed and altitude control were emphasized above all other attributes (eg., flight path trajectory). It will be useful to determine whether the current 
principles for ideal instrument scanning are generalizable to different flight scenarios and maneuvers. A general model for ideal instrument scanning would be useful for training purposes as well as interface design.

\section{References}

1. Yang, J.H., Kennedy, Q., Sullivan, J., Fricker, R.D.: Pilot performance: Assessing how scan patterns navigational assessments vary by flight expertise. Aviation, Space, and Environmental Medicine 84(2), 116-124 (2013)

2. Anders, G.: Pilot's attention allocation during approach and landing- Eye-and headtracking research in an A 330 full flight simulator. Focusing Attention on Aviation Safety (2001)

3. Nieuwenhuizen, F., Jump, M., Perfect, P., White, M., Padfield, G., Floreano, D., Schill, F., Zufferey, J., Fua, P., Bouabdallah, S., et al.: mycopter-enabling technologies for personal aerial transportation systems. In: Proceeding of the 3rd International HELI World Conference 2011, pp. 1-8 (2011)

4. MyCopter: Enabling Technologies for Personal Aerial Transport Systems, http://www.mycopter.eu

5. Perry, A.: The flightgear flight simulator. In: 2004 USENIX Annual Technical Conference, Boston, MA (2004)

6. Wetzel, P.A., Anderson, G.M., Barelka, B.A.: Instructor use of eye position based feedback for pilot training. In: Proceedings of the Human Factors and Ergonomics Society Annual Meeting, vol. 42, pp. 1388-1392. SAGE Publications (1998) 